\title{
Autism and schizophrenia: one, two or many disorders? ${ }^{\dagger}$
}

\author{
Stephen J. Wood
}

\section{Summary}

Autism and psychotic disorders such as schizophrenia co-occur more frequently than would be expected by chance alone. Exactly why this should be remains unclear, but a better understanding would have important implications for diagnosis, treatment and for biological explanations of both conditions.

\author{
Declaration of interest \\ None.
}

\section{Copyright and usage}

(c) The Royal College of Psychiatrists 2017.
Stephen Wood is Professor of Clinical and Translational Neuroscience at Orygen, The National Centre for Excellence in Youth Mental Health, in Melbourne, and at the Centre for Youth Mental Health, University of Melbourne (Australia). He is also Professor of Adolescent Development and Mental Health at the School of Psychology, University of Birmingham (UK).

The relationship between autism and schizophrenia is complex and has undergone significant rethinking over the past 70 years. In the mid-twentieth century, the two disorders were actually conceptualised as one, with Bleuler considering autism a central feature of schizophrenia ${ }^{1}$ and Bender viewing it as the childhood onset of the illness. ${ }^{2}$ From the early 1970s, though, the two began to be regarded as separate conditions. ${ }^{3}$ In many ways this is justified - the age at onset being the most obvious example where the disorders differ. However, it is now widely accepted that there is significant overlap between the two conditions, in their genetic underpinnings, epidemiological similarities and the high rates of co-occurrence. ${ }^{4}$

\section{Presentation of comorbid autism and psychotic disorders}

This overlap is explored in some detail in a report from Larson and colleagues, in this issue of the BJPsych. ${ }^{5}$ By investigating people presenting with co-occurring autism spectrum disorders (ASD) and psychotic illness, they aimed to better understand the relationship between the two disorders. Key to their study is the direct comparison of patients with ASD and psychosis (ASD-P group) with patients with only psychosis, and with only autism (ASD-NP group). This showed that a diagnosis of schizophrenia was significantly less common in the ASD-P group compared with those with psychotic illness alone. This was reflected in a higher rate of a psychosis not otherwise specified (NOS) diagnosis (44\% of all those in the ASD-P group) and prominent affective symptoms. Furthermore, those with ASD-P had fewer stereotyped, repetitive or restrictive interests/behaviours than those in the ASD-NP group, and were less likely to be female (although in this respect, the ASD-NP group does not seem representative of the autism spectrum generally; here the male-tofemale ratio was equal, whereas the literature commonly shows males at least three times as likely to have an autism spectrum diagnosis ${ }^{6}$ ).

Overall then, this work suggests that the presentation of comorbid ASD and psychotic disorder is different from the

'See pp. 269-275, this issue. usual presentation of the two disorders alone. But what does this mean for our understanding of the relationship between these two conditions? In 2015, Chisholm and colleagues published a review of autism and schizophrenia, including an examination of various models of their co-occurrence. ${ }^{4}$ The findings from Larson et al seem to provide qualified support for the 'independence' model. Such a model proposes that when two disorders co-occur, the resultant condition is an independent, distinct nosological entity. For example, people who experience periods of depression and periods of mania are not considered to have two co-occurring conditions but instead a third separate one: bipolar disorder. In the case of autism and psychosis, this third condition would seem to be characterised by non-schizophrenia presentation, prominent affective symptoms and few (or fewer) stereotypical behaviours. As the authors point out themselves, this suggests that in fact the overlap between autism and schizophrenia specifically is rather limited. Whether there would be clinical utility in such a diagnostic revision remains to be determined.

However, there is only very limited evidence from other work for the independence model, and it is more likely that these current findings buttress a 'multiple overlapping aetiologies' model. This has similarities with the independence model, but emphasises the heterogeneity of both autism and schizophrenia. It is quite feasible for there to be many distinct clinical syndromes arising from shared and unique genetic and environmental risk factors, which can be clustered into two major groups ('the autisms' and 'the schizophrenias'). Because there are overlapping features and multiple causal pathways, co-occurrence is to be expected.

\section{Conclusions}

What then can be done to improve our understanding? As Larson et al note, their sample may not be representative, which would affect our understanding of the phenomenology associated with comorbidity. Therefore, a more systematic assessment of the prevalence of co-occurring autism and psychotic illness is required. Ideally this would be accompanied by measurement of autistic and schizotypal traits, along with their stability over time. Furthermore, qualitative work to better understand the particular issues experienced by those with ASD when they come into contact with mental health services could result in significant improvements to patient care. Finally, we need to be more open to the complexities of clinical presentation, particularly as researchers. If, as seems possible, autism and psychosis traits interact to moderate perspective-taking impairments, ${ }^{7}$ or affect 
activation of the temporoparietal junction, ${ }^{8}$ then we will need to pay more than lip service to building multidimensional models of psychopathology.

Stephen J. Wood, MA(Cantab), PhD, Orygen, The National Centre for Excellence in Youth Mental Health, 35 Poplar Road, Parkville, Victoria 3052, Australia. Email: stephen.wood@orygen.org.au

First received 23 Nov 2016, accepted 19 Jan 2017

\section{References}

1 Bleuler E. Dementia Praecox or the Group of Schizophrenias. International Universities Press, 1950

2 Bender L. Childhood schizophrenia. Am J Orthopsychiatry 1947; 17: 40-56.
3 Rutter M. Childhood schizophrenia reconsidered. J Autism Dev Disord 1972; 2: $315-37$.

4 Chisholm K, Lin A, Abu-Akel A, Wood SJ. The association between autism and schizophrenia spectrum disorders: a review of eight alternate models of co-occurrence. Neurosci Biobehav Rev 2015; 55: 173-83.

5 Larson FV, Wagner AP, Jones PB, Tantam D, Lai M-C, Baron-Cohen S, et al. Psychosis in autism: a comparison of the features of both conditions in a dually-affected cohort. Br J Psychiatry 2017: 210: 269-75.

6 Werling DM, Geschwind DH. Sex differences in autism spectrum disorders. Curr Opin Neurol 2013; 26: 146-53.

7 Abu-Akel A, Wood SJ, Hansen PC, Apperly IA. Perspective-taking abilities in the balance between autism tendencies and psychosis proneness. Proc Bio Sci 2015; 282: 20150563.

8 Abu-Akel A, Apperly IA, Wood SJ, Hansen PC. Autism and psychosis expressions diametrically modulate the right temporoparietal junction. Soc Neurosci 2016; June 3 (Epub ahead of print).

\section{psychiatry in pictures}

\section{Simba Muzira}

Sara Muzira

These images are from an exhibition of Simba Muzira's work titled 'Doing it again'. The exhibition runs at the Long Gallery, Maudsley Hospital, from 1 February to 1 May 2017. Simba was a patient at the Maudsley on several occasions from 2012 unti his death in 2015, aged 32. For more information, see www.slam.nhs.uk/about-us/art-and-history/maudsley-long-gallery
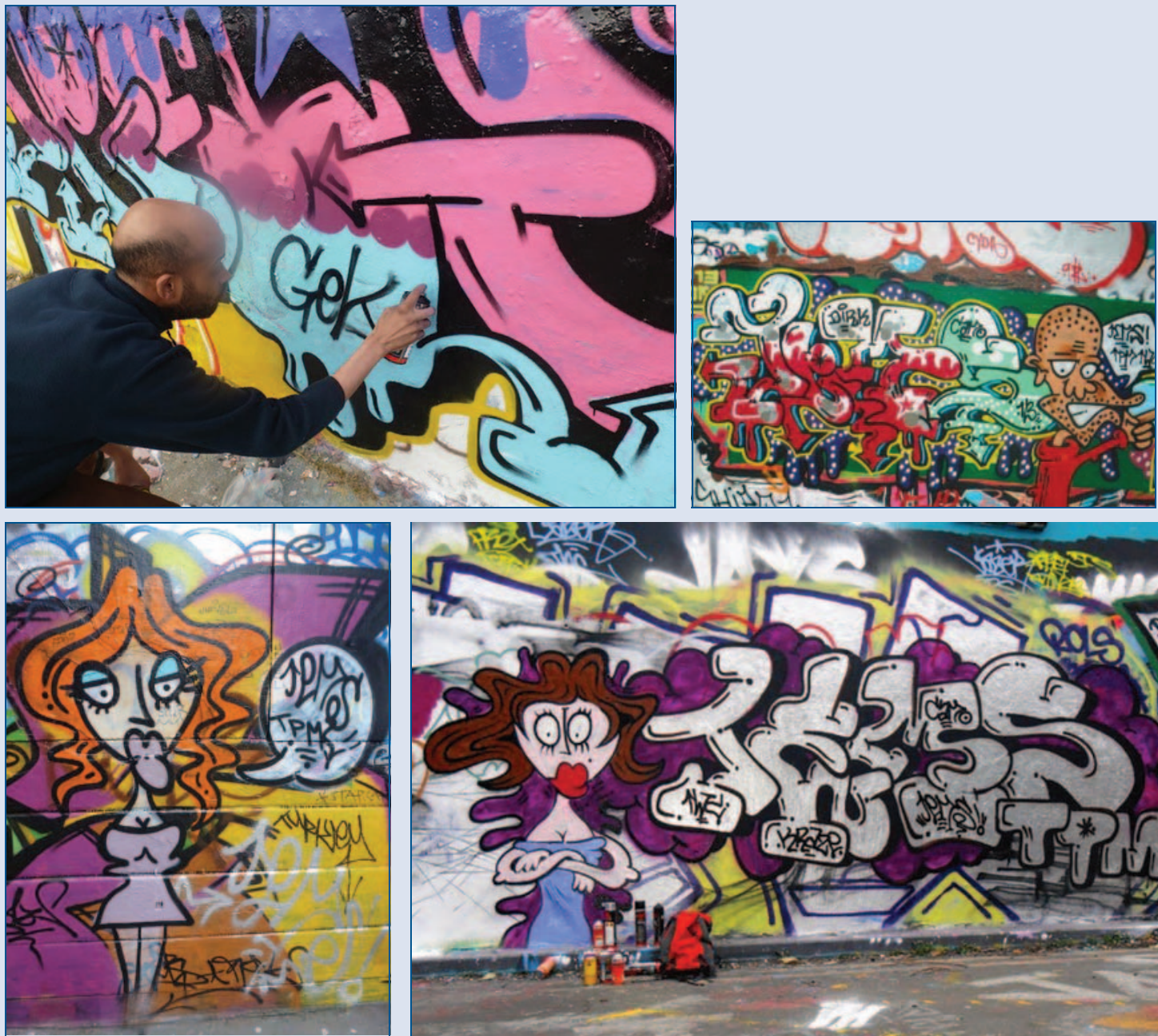